\title{
A Method to Reduce Interference by Sucrose in the Detection of Thiobarbituric Acid-Reactive Substances ${ }^{1}$
}

\author{
Marshal Shlafer and Beverly M. Shepard \\ Department of Pharmacology: Lniversity of Michigan Medical School, Ann Arhor, Michigan 48109
}

Received May 9. 1983

\begin{abstract}
A thiobarbituric acid (TBA) reaction for measuring lipid peroxidation products was evaluated for interference by several ingredients commonly used in solutions to prepare or analyze tissue homogenates or subcellular organelles. These included sucrose (up to $100 \mathrm{~mm}$ final concentration in the assay medium). Tris-maleate (up to $40 \mathrm{mM}$ ), imidazole (up to $20 \mathrm{mM}$ ), inorganic phosphate (up to $10 \mathrm{~mm}$ ), and 4-morpholinepropanesulfonic acid (up to $20 \mathrm{~mm}$ ). When the samples were heated at $95^{\circ} \mathrm{C}$ as recommended in some procedures, only sucrose significantly affected color development. Sucrose concentrations as low as $10 \mathrm{~mm}$ significantly increased absorbance at 532 $\mathrm{nm}$ of aqueous tetramethoxypropane (TMP) standards, and so the assay could not be applied reliably to tissue samples prepared in sucrose. Sucrose interference was only partially reduced by subsequent organic extraction ( $n$-hutanol plus pyridine), with measured absorbances remaining significantly grcater $(50-100 \%)$ than sucrose-free controls at sucrose concentrations of $20 \mathrm{~mm}$ or more. Modifying the assay to include sucrose in blanks and IMP standards failed to adequately correct for interference when the absorbance of unextracted (aqueous) solutions was measured. Further modification by adding sucrose to blanks and TMP standards, followed by butanolpyridine extraction, gave standard curves that were lintear. through the origin, and had slopes equivalent to those of sucrose-free standards. This modification enabled almost complete recovery (average $2 \%$ error) of known amounts of TMP added to aliquots of tissue homogenates containing amounts of sucrose that otherwise significantly interfered. Also. with the modified method the cortent of TBA-reactive substances in tissues homogenized in sucrose was found to be not significantly different from that measured in tissues homogenized in a noninterfering substance, $\mathrm{KCl}$.

KEY WORDS: lipid peroxidation; thiobarbituric acid; malonaldehyde; methods.
\end{abstract}

The importance of lipid peroxidation in the alteration of many biological systems by various pathological states and drugs is becoming increasingly apparent. Assays involving thiobarbituric acid (TBA) ${ }^{2}$ are widely used to obtain evidence of peroxidation because they are generally quick, sensitive, and require little instrumentation other than a spectrophotom. eter. Nevertheless there are problems with TBA-based assays: it is not appropriate for measuring lipid peroxidation products in vivo

\footnotetext{
${ }^{1}$ Supported by NIH Grant HL-29499-01.

2 Abbreviations used: TBA, thiobarbituric acid; TMP, tetramethoxypropane; Mops, 4-morpholinepropanesulfonic acid; SDS, sodium dodecyl sulfate.
}

(1), a variety of substances interfere with the reaction (2-8), and the results are dependent upon assay conditions and the lipid composition of the sample $(4,9-12)$. While the best resolution of these problems can be achieved using high-performance liquid chromatography, that technique is more time-consuming. more costly, and not available to many investigators.

While adapting a TBA assay to our research we found that some media gave clearly inappropriate and inaccurate results when samples were incubated at $95^{\circ} \mathrm{C}$ as recommended by Ohkawa and colleagues (9). Upon systematically investigating the ingredients in our solutions we found that sucrose, one of the most 
common ingredients in tissue and organelle isolation media, at concentrations as low as $10 \mathrm{~mm}$, doubled the absorbance of tetramethoxypropane (TMP) standards. Absorbances of standards containing $100 \mathrm{mM}$ sucrose gave fivefold overestimates of the actual TMP content. Butanol-pyridine extraction of the reactants only partially reduced the interference. We subsequently found that interference could be reduced almost completely by including sucrose in blanks and TMP standards, followed by organic extraction.

\section{MATERIALS AND METHODS}

Tetramethoxypropane standards. Malonaldehyde bis(dimethyl acetal) [1,1,3,3-tetramethoxypropane; TMP] was purchased from Aldrich (Milwaukee, Wisc.). One millimole was weighed into a 1-liter volumetric flask, to which approximately $500 \mathrm{ml}$ water and $1 \mathrm{ml}$ of $1 \mathrm{~N} \mathrm{HCl}$ were added. The flask was stoppered, heated at $50^{\circ} \mathrm{C}$ for $60 \mathrm{~min}$, and cooled to $25^{\circ} \mathrm{C}$, and the volume was adjusted to 1000 $\mathrm{ml}$. This solution was kept at $4^{\circ} \mathrm{C}$ in the dark for up to 1 month with no detectable change of absorbance of standard aliquots when measured as described below. Water used for all aqueous solutions was double-distilled in glass and further purified by a Hydro-Services (Durham, N. C.) OA-18 organic adsorbent column and two DM-18M mixed-bed ion-exchange columns.

Evaluating interference with a published method. We first evaluated whether several commonly used ingredients in preparative media affected the absorbance of TMP standards ( 1 to $10 \mathrm{nmol}$ ) when assayed as described recently by Ohkawa et al. (9). Various amounts of the following stock solutions were tested at final concentrations indicated under Results: sucrose (analytical-reagent grade; 0.88; M; Mallinckrodt); 4-morpholinepropanesulfonic acid (Mops; $0.15 \mathrm{M}$; Sigma); Trismaleate $(0.2 \mathrm{M}$; Trizma maleate. Sigma): imidazole (0.09 M; Sigma); and potassium phosphate buffer $(0.05 \mathrm{M}, \mathrm{pH} 8.0$, monobasic and dibasic salts obtained from Sigma). None of these additives changed the $\mathrm{pH}$ of the assay medium. To avoid changing the assay volume, appropriate volumes of water were omitted from tubes receiving an additive. The absorbances of the aqueous standards and their butanol-pyridine extracts were measured at 532 $n m$ against blanks that contained neither TMP nor additive. The absorbances of standards containing additive were compared statistically to those of additive-free standards that contained the same amount of TMP.

With the assay conducted as above (9), we found that only sucrose caused significant interference (see Results). We subsequently developed the following modification for use when the TB $\Lambda$ assay is applied to biologic samples that are prepared in a solution containing sucrose.

Modified TBA assay for samples containing sucrose standards and hlank. Standards were prepared to contain 1 to $10 \mathrm{nmol}$ TMP. To the aliquot of TMP were added $200 \mu \mathrm{l}$ of $8.1 \%$ $(\mathrm{w} / \mathrm{v})$ sodium dodecyl sulfate (SDS); $1.5 \mathrm{ml}$ of $20 \%(\mathrm{v} / \mathrm{v})$ acetic acid (previously adjusted to $\mathrm{pH} 3.5$ by adding a small volume of $5 \mathrm{~N}$ $\mathrm{NaOH}$ ); sucrose-containing solution equal in volume and composition to that which will be used for analysis of the biologic sample; and sufficient distilied water to give a volume of $2.5 \mathrm{ml}$. To this was added $1.5 \mathrm{ml}$ of fresh $0.8 \%(\mathrm{w} / \mathrm{v})$ (TBA (Eastman Organic Chemicals). The tubes were vortexed well. The blank contained all of the above except TMP.

Biologic samples. To assay the content of TBA-reactive substances in biologic samples, duplicate or triplicate tubes were prepared as described above using 100 - to $400-\mu l$ aliquots of sample in lieu of TMP and adjusting the amount of water added to maintain a final volume of $2.5 \mathrm{ml}$ before adding TBA. If the amount of TBA-reactive substances will be measured in several different volumes of sample (that is, in the presence of different sucrose concentrations), it may be necessary to prepare separate sets of appropriately corrected blanks and standards for each amount of sucrose in the assay tubes.

The tubes were covered with a glass marble and heated at $95^{\circ} \mathrm{C}$ for $60 \mathrm{~min}$. Adherence to 
the time and temperature guidelines is imporlant for reproducibility. The samples were then cooled to room temperature. Five milliliters of a mixture of $n$-butanol and pyridine $(15: 1, v / v)$ was added to each tube, the tubes were vigorously mixed again and centrifuged for 10 min at approximately $2200 \mathrm{~g}$, and the organic phase was carefully removed with a pipet and transferred to a new set of glass tubes. The absorbances of the organic extracts were measured at $532 \mathrm{~nm}$ against the extract of the blank.

Preparation and assay of tissue homogenates. Samples of fresh rabbit heart, lung, liver, or skeletal muscle were homogenized (Tekmar Tissuemizer, Tekmar Instruments, Cincinnati) in $4 \mathrm{vol}(\mathrm{ml} / \mathrm{g}$ tissue wet wt) of medium containing $0.25 \mathrm{M}$ sucrose and $5 \mathrm{~mm}$ Tris-maleate ( $\mathrm{pH} 6.8$ at $4^{\circ} \mathrm{C}$ ). More cold medium was added to give $10 \mathrm{ml}$ homogenate $/ \mathrm{g}$ tissue wet $\mathrm{wt}$. The modified TBA assay was performed on duplicate or triplicate samples (up to $400 \mu 1$ ) of each homogenate as described above. The absorbances of the samples were determined at $532 \mathrm{~nm}$ using blanks and TMP standards corrected by adding volumes of the homogenizing medium equal to that used for the homogenate samples.

In other experiments samples of fresh rabbit liver were quickly divided into two portions; one was placed in $1.15 \%(\mathrm{w} / \mathrm{v}) \mathrm{KCl}$ as done by Ohkawa of al. (9), and the other was placed in the sucrose-containing medium described above. The samples were homogenized and diluted as described above, and the amount of TBA-reactive substances of samples prepared in $\mathrm{KCl}$ (uncorrected, as $\mathrm{KCl}$ did not interfere) was compared by analysis of variance to that of samples in sucrose assayed with the modified protocol. TMP standard curves made in the presence of $\mathrm{KCl}$ or sucrose were also compared.

For the data reported below the assays were usually run on separate days in duplicate or triplicate. To characterize further interference by sucrose, and the applicability of the modified protocol, we usually used final sugar concentrations around $20 \mathrm{mM}$, since this ap- proximated the concentration reached when $300-\mu 1$ aliquots of a $0.25 \mathrm{M}$ sucrose-containing medium were assayed under the conditions noted above.

\section{RESULTS}

\section{Interference by Additives with Lincorrected Blanks and Standards}

Table 1 shows that neither Mops (up to a final concentration of $20 \mathrm{~mm}$ ). Tris-maleate (up to $40 \mathrm{~mm}$ ), imidazole (up to $20 \mathrm{~mm}$ ) nor inorganic phosphate (up to $10 \mathrm{~mm}$ ) affected color development of 1 to $10 \mathrm{nmol}$ TMP standards measured at $532 \mathrm{~nm}$ (data from the 4 nmol standards are presented in the table), whether color was measured in the original aqueous phase or after extraction with butanol plus pyridine. Mops slightly inhibited color development, but the effect was not statistically significant. In contrast, sucrose significantly interfered due to the development of a yellow product. Final sucrose concentrations of $10 \mathrm{mM}$ or more significantly increased the absorbance of aqueous (unextracted) samples at $532 \mathrm{~nm}$, giving apparent TMP contents two to five times higher than what was actually in the sample. Extracting the sucrose-containing samples with butanol and pyridine as described by Ohkawa et al. (9) reduced the interference by sucrose somewhat, but, based on $t$ tests comparing the absorbances of TMP standards with and without sucrose, only extracted samples containing $10 \mathrm{~mm}$ sucrose or less had absorbances that were not significantly different from sucrose-free controls. With sucrose concentrations of $20 \mathrm{~mm}$ or more butanol-pyridine extraction failed to adequately reduce interference by sucrose.

The interference produced by sucrose was affected by incubation time and temperature. We found in preliminary experiments that incubating the samples at $95^{\circ} \mathrm{C}$ for more than $70 \mathrm{~min}$ or so obviously deepened the yellow color further, whereas incubating samples below approximately $89^{\circ} \mathrm{C}$ for $60 \mathrm{~min}$ appeared to reduce color development. However, we did not attempt to quantify the time or tem- 
TABLE 1

EFFECTS OF COMMON LABORATORY MFDIa COMPONENTS ON APPARENT TMP CONTENT OF 4-nmol TMP STANDARDS ESTIMATED BY THE TBA REACTION WITH. OUT ADJUSTMENT OF BLANKS AND STANDARDS

\begin{tabular}{|c|c|c|}
\hline \multirow[b]{2}{*}{$\begin{array}{c}\text { Addition } \\
(\mathrm{mM})\end{array}$} & \multicolumn{2}{|c|}{ Apparent TMP content (nmol) } \\
\hline & $\begin{array}{l}\text { Aqueous } \\
\text { phase }\end{array}$ & $\begin{array}{c}\text { Butanol:pyridine } \\
\text { extract }\end{array}$ \\
\hline None & 4.0 & 4.0 \\
\hline \multicolumn{3}{|l|}{ Sucrose $^{a}$} \\
\hline 1 & $5.18 \pm 0.91$ & $4.40 \pm 0.56$ \\
\hline 10 & $8.27 \pm 0.54^{*}$ & $5.04 \pm 0.42$ \\
\hline 20 & $11.92 \pm 0.51^{*}$ & $6.40 \pm 0.60^{*}$ \\
\hline 40 & $15.48 \pm 1.26^{*}$ & $6.92 \pm 0.60^{*}$ \\
\hline 80 & $19.34 \pm 1.30^{*}$ & $8.72 \pm 0.60^{*}$ \\
\hline 100 & $20.34 \pm 1.50^{*}$ & $8.86 \pm 0.67^{*}$ \\
\hline \multicolumn{3}{|l|}{ Mops } \\
\hline 1 & $3.76 \pm 0.21$ & $3.74 \pm 0.28$ \\
\hline 10 & $4.08 \pm 0.15$ & $3.46 \pm 0.33$ \\
\hline 20 & $3.82 \pm 0.14$ & $3.58 \pm 0.26$ \\
\hline \multicolumn{3}{|c|}{ Tris-maleate } \\
\hline 1 & $3.84 \pm 0.12$ & $3.76 \pm 0.43$ \\
\hline 10 & $4.21 \pm 0.12$ & $3.62 \pm 0.22$ \\
\hline 20 & $4.02 \pm 0.19$ & $4.32 \pm 0.46$ \\
\hline 40 & $4.02 \pm 0.24$ & $3.84 \pm 0.24$ \\
\hline \multicolumn{3}{|l|}{ Phosphate } \\
\hline 1 & $3.94 \pm 0.21$ & $3.90 \pm 0.22$ \\
\hline 5 & $4.06 \pm 0.29$ & $3.42 \pm 0.24$ \\
\hline 10 & $3.82 \pm 0.18$ & $4.18 \pm 0.43$ \\
\hline \multicolumn{3}{|l|}{ Imidazole } \\
\hline 1 & $3.94 \pm 0.13$ & $3.92 \pm 0.29$ \\
\hline 10 & $3.98 \pm 0.17$ & $4.40 \pm 0.63$ \\
\hline 20 & $4.00 \pm 0.19$ & $3.64 \pm 0.23$ \\
\hline
\end{tabular}

Note. The spectrophotometric assay was conducted as described by Ohkawa et al. (9). Standards were run with $4 \mathrm{nmol}$ TMP in a total volume of $4 \mathrm{ml}$; blanks were prepared without including the test substance.

a The millimolar concentration indicates the final concentration of additive in the complete reaction medium. Values marked with an asterisk $\left({ }^{*}\right)$ had absorbances that were significantly different $(P<0.05)$ from that of 4 -nmol standards that did not contain any potentially interfering substance.

perature dependency of these changes since our objective was to evaluate sucrose interference under specified conditions. It is nevertheless clear that when the assay involves relatively high concentrations of sucrose, strict control of time and temperature is necessary.

Figure 1 shows the relationship between absorbance at $532 \mathrm{~nm}$ and the amount of TMP. We found a linear relationship up to at least $10 \mathrm{nmol}$ TMP in aqueous standards and their organic extracts, without or with $20 \mathrm{~mm}$ sucrose (final concentration in assay medium). In the absence of sucrose (circles) the standard curves for the aqueous phase and the organic extracts were linear $(r=0.961$ and 0.944 , respectively), but, based on analysis of the regression lines, their slopes were significantly different $(P<0.001)$. Additionally, based on regression analysis the line for the aqueous standards had a $y$ intercept that was close to, but not through, the origin.

\section{Effects of Correcting Blank and Standards with Sucrose, Followed by Organic Extraction (Modified Method)}

When we added sucrose to both blanks and standards (open squares) we found that, although the absorbances of the aqueous (unex-



FIG. 1. Standard curves for tetramethoxypropane in aqueous solutions (AQ: open symbols) and butanol-pyridine extracts (BPE; filled symbols) without (circles; Control) or with (squares) $20 \mathrm{~mm}$ sucrose (final concentration). For determinations with sucrose, the sugar was added to both the TMP standards and the blank. Data points are arithmetic means \pm I SEM, and lines were fitted by linear regression analysis. 
tracted) standards were linear $(r=0.900)$ with respect to TMP content, the line did not pass through the origin. The slope of this line was also significantly different $(P<0.02)$ from that of the line obtained with aqueous, sucrosefree standards.

When the aqueous sucrose-containing standards were extracted with butanol-pyridine we obtained a linear $(r=0.944)$ relationship between absorbance and TMP content that passed through the origin and had a slope that was not significantly different from the sucrose-free organic extract line.

\section{Absorbance Spectra with and without TMP or Sucrose}

Spectra of blanks and TMP standards, with or without sucrose ( 10 to $80 \mathrm{mM})$, were obtained from aqueous and extracted samples using a Cary Model 219 spectrophotometer. Figure 2 shows spectra of unextracted $4 \mathrm{nmol}$ TMP standards reacted with TBA (A), $25 \mathrm{~mm}$ sucrose reacted with TBA in the absence of TMP $((B)$; a sucrose-containing blank), and 4

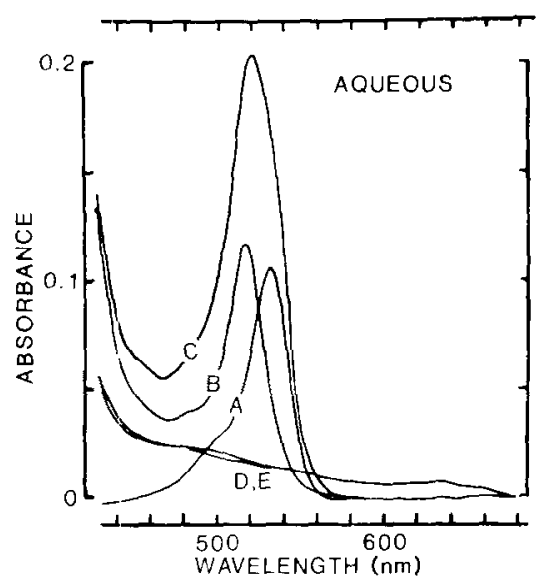

FIG. 2. Spectra of uncorrected aqueous assay reactants containing TMP alone (A; $4 \mathrm{nmol}$ ), sucrose alone (B; 25 $\mathrm{mM}$ ), or TMP plus sucrose $(\mathrm{C})$. The reference cuvette contained no sucrose or TBA. Absorbances of liver homogenate made in $\mathrm{KCl}$ and reacted with SDS and acetic acid. without TBA and TMP, in the presence (D) or absence (E) of added sucrose (19 $\mathrm{mM}$ ). suggest that incubation of tissue with sucrose does not produce a colored product with appreciable absorption at $532 \mathrm{~nm}$. nmol TMP plus $25 \mathrm{mM}$ sucrose reacted with IBA (C). IMP alone produced a single peak at 531-532 nm. The peak absorbance for sucrose in the absence of TMP was at 516-517 $\mathrm{nm}$. but absorbance at $532 \mathrm{~nm}$ was also appreciable. The peak with TMP in the presence of sucrose was at $520 \mathrm{~nm}$. and absorbance was nearly double that measured in the other tubes.

Extracting the 4-ml aqueous aliquots with $5 \mathrm{ml}$ butanol-pyridine (Fig. 3) shifted the peak absorbance for TMP alone (A) slightly to 532$534 \mathrm{~nm}$. Extracting sucrose-containing (TMPfree) samples (Fig. 3B) markedly reduced absorbance at wavelengths of $500 \mathrm{~nm}$ or more, and caused the appearance of a distinct peak at $450 \mathrm{~nm}$. Extracted standards of $4 \mathrm{nmol}$ TMP in the presence of $25 \mathrm{~mm}$ sucrose (Fig. $3 C$ ) had a peak absorbance at $532-533 \mathrm{~nm}$, but the maximum absorbance of this combination was still significantly higher than that of sucrose-free TMP standards, indicating that organic extraction alone was insufficient to eliminate interference. When the absorbance of extracted TMP-sucrose was measured against a corrected (sucrose-containing) blank, maximum absorbance at $532-533 \mathrm{~nm}$ was identical to that obtained with sucrose-free TMP standards (e.g., Fig. 3A), indicating that the modified method caused a significant decrease of interference. An absorbance peak at $450 \mathrm{~nm}$ remained.

\section{Effects of Modified Protocol on Recovery of TMP Added to Homogenate}

The modified procedure allowed us to detect virtually all of a known amount of TMP added to tissue homogenate samples prepared in 0.25 $M$ sucrose. The mean absorbance differences [(homogenate plus TMP) - (homogenate without TMP)] were converted to nanomoles of TMP from the standard curve and compared to the amount of TMP added $(4 \mathrm{nmol})$ using chi-square analysis. For example, liver homogenate samples $(200 \mu \mathrm{l})$ had $5.28 \pm 0.62$ nmol TBA-reactive substances per aliquot $(N$ $=11$; mean $\pm \mathrm{SE}$ ). The mean difference between those samples and duplicate aliquots to 


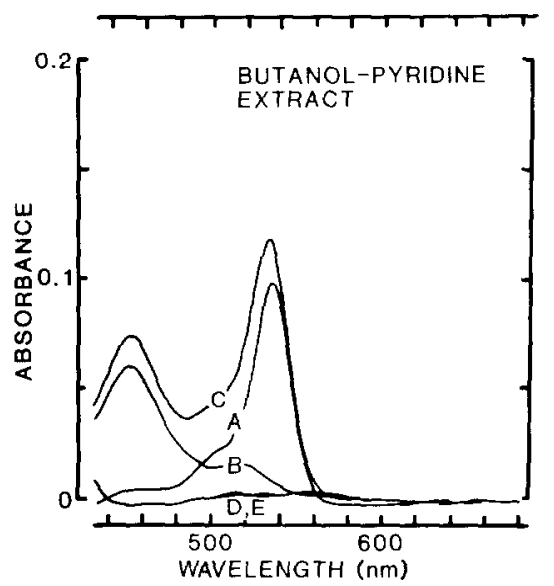

FIG. 3. Samples shown in Fig. 2 were extracted with butanol-pyridine and scanned again against a sucrosefree reference (blank). Organic extraction reduced overall absorbance of tubes containing only sucrose (B), sucrose plus TMP $(C)$, or liver tissue incubated with (D) or without (E) $19 \mathrm{mM}$ sucrose.

which $4 \mathrm{nmol}$ TMP was added was $4.08 \mathrm{nmol}$ (determined from triplicate measurements of the 11 samples). When the differences from each experiment were averaged, the overestimate was $1.9 \%$. Based on chi-square analysis, the observed values were not significantly different $(P>0.25)$ from the expected values. Similar results were obtained with heart, lung. and skeletal muscle.

\section{Effects of Slight Differences of Sucrose Concentrations in Standards and Biologic Samples}

When applying the modified assay to biologic samples containing relatively large amounts of water (e.g., to obtain the data reported in the preceding paragraph), the sucrose concentration in the final sample will be fractionally lower than that of the medium before tissue was added. Furthermore, if a volume of the tissue-free medium equal to that of the biologic sample is used to adjust the blank and standards, their final sucrose concentrations would be fractionally higher than those of the homogenate samples. Since each sample would have relatively less sucrose to interfere, this might cause underestimation of the amount of TBA-reactive substances in the sample. For example, homogenizing $1 \mathrm{~g}$ of tissue containing $80 \%$ water in $9 \mathrm{ml}$ of solution containing $0.25 \mathrm{M}$ sucrose dilutes the sucrose to a final concentration of $0.23 \mathrm{M}$. This disparity could be important when a given volume of homogenate $(0.23 \mathrm{M}$ with respect to sucrose) is assayed using blanks and standards prepared by adding homogenate containing $0.25 \mathrm{M}$ sucrose. We therefore evaluated whether blanks and standards could be adequately corrected by simply adding an aliquot of sample-free, sucrose-containing medium, ignoring the dilution factor, or whether further correction of blanks and standards to account for sample water was necessary.

TMP standards ( $4 \mathrm{nmol} ; N=10$ ) containing $22 \mathrm{mM}$ sucrose (final concentration in assay) were prepared along with a blank containing $22 \mathrm{~mm}$ sucrose. To mimic dilution of sucrose by tissue water. $4 \mathrm{nmol}$ TMP standards were prepared to contain approximately 10 to $30 \%$ less sucrose $(20,18$, and $15 \mathrm{~mm}$, respectively), and used as if they were tissue samples. Their absorbances were measured at $532 \mathrm{~nm}$ against only the blank containing $22 \mathrm{mM}$ sucrose. Average absorbances as well as the apparent TMP contents were computed and compared statistically to the absorbances of the $4 \mathrm{nmol}$ standards in $22 \mathrm{~mm}$ sucrose. The apparent amounts of TMP (means \pm 1 SE) were 3.96 $\pm 0.1 \mathrm{nmol}$ in the $20 \mathrm{~mm}$ sucrose standards (mean underestimate $1.0 \%$ from the expected value of $4.00 \mathrm{nmol}$ ); $4.00 \pm 0.10 \mathrm{nmol}$ in the $18 \mathrm{~mm}$ sucrose standards (mean difference $0 \%$ ); and $3.94 \pm 0.04 \mathrm{nmol}$ in the $15 \mathrm{~mm}$ standards ( $1.4 \%$ mean underestimate). Thus, unless relatively large amounts of water-containing sample are prepared in small volumes of sucrose-containing medium for subsequent application of the modified TBA assay, it may be acceptable to ignore dilution of sucrose by sample water.

In contrast to the above possibility, unless there is a major methodologic error, it is much less likely that biologic sample tubes would have an appreciably greater final sucrose concentration than the corrected blank and standards. which would overestimate the amount 
of TBA-reactive substance in the sample. Nevertheless, using the method of comparison noted above, we prepared a series of $4 \mathrm{nmol}$ TMP standards in assay tubes to which additional sucrose was added to give concentrations of 24 and $26 \mathrm{mM}$, and determined their apparent TMP contents using the blank that contained only $22 \mathrm{~mm}$ sucrose. The apparent TMP content of standards made with $24 \mathrm{~mm}$ sucrose was $4.06 \pm 0.05 \mathrm{nmol}(1.3 \%$ mean overestimate), while those made in $26 \mathrm{~mm}$ sucrose had an apparent content of $4.19 \pm 0.04$ nmol ( $4.7 \%$ overestimate). Thus, the modified method is less tolerant of errors in this direction, although the likelihood of such an error is low.

\section{Comparison of $\mathrm{KCl}$ and Sucrose as Media for the Determination of Tissue TBA- Reactive Substances}

We compared the results of assays of homogenates prepared with $\mathrm{KCl}(9)$ to those obtained with the modified assay of a homogenate of the same tissue in sucrose. The sucrose-containing assay tubes had final sucrose concentrations ranging from $11.5 \mathrm{~mm}$ (200 $\mu \mathrm{l}$ of sample and assuming $80 \%$ water content) to $22.5 \mathrm{~mm}(400-\mu 1$ samples). The amounts of TBA-reactive substances in 200-, 300-, and 400- $\mu$ l samples prepared in $\mathrm{KCl}$ and extracted with butanol-pyridine were $2.22 \pm 0.20,3.36 \pm 0.32$, and $4.54 \pm 0.35$ nmol, respectively (mean $\pm \mathrm{SE} ; N=9$ ). Those of samples prepared in sucrose, extracted, and measured against appropriate blanks and standards were $2.19 \pm 0.06,3.46 \pm 0.19$, and $4.44 \pm 0.20 \mathrm{nmol}$, respectively. Based on $t$ tests, values obtained with sucrose were not significantly different from those obtained with $\mathrm{KCl}$. When the amounts of TBA-reactive substances were expressed in terms of sample protein (Biuret), the overall mean of 200-. $300-$, and $400-\mu 1$ samples prepared in sucrose was $0.49 \pm 0.02 \mathrm{nmol} / \mathrm{mg}$. and that for the samples in $\mathrm{KCl}$ was $0.51 \pm 0.02 \mathrm{nmol} / \mathrm{mg}$ (no significant difference). The slopes and intercepts of the regression lines relating sample volume to the amount of TBA-reactive substance in the sample (either regular linear regression or regression for lines through the origin) were not significantly different when comparing samples prepared in sucrose to those prepared in $\mathrm{KCl}$. Also, there were no significant differences in the absorbances of TMP standards from the sucrose or $\mathrm{KCl}$ experiments.

We recorded absorbance spectra (Figs. 2, 3 ) of liver prepared in $\mathrm{KCl}$ and incubated for $60 \mathrm{~min}$ at $95^{\circ} \mathrm{C}$ with acetic acid, SDS, and various amounts of sucrose (or water in controls) to determine whether liver homogenates plus sucrose formed a product with significant absorbance at wavelengths close to those for the TBA-TMP product with sucrose. The absorbance of aqueous samples (Fig. 2; at 532 $\mathrm{nm}$ was low. At all wavelengths measured there was no difference between liver homogenates incubated with (Fig. 2D) or without (Fig. 2E) sucrose. Butanol-pyridine extraction of these samples (Figs. 3D, E) reduced absorbance almost completely. These data suggest that sucrose docs not react with some liver tissue component which might be erroneously detected as a TBA-reactive substance.

\section{DISCUSSION}

The low sucrose concentrations that interfere can be easily reached in analytical procedures that assay small volumes of samples prepared in media having sucrose concentrations around $0.25 \mathrm{M}$, as is commonly used to maintain osmotic equilibrium in in vitro experiments. It is conceivable that an investigator might use sample volumes even larger than we used, producing final sucrose concentrations greater than $20 \mathrm{~mm}$, in an attempt to determine whether lipid peroxidation has occurred in very dilute systems or systems that may be mimimally perturbed by peroxidative processes. Media containing sucrose concentrations much higher than $0.25 \mathrm{M}$ are routinely used for centrifugation, so the chance of interference is also great in that setting. Our data indicate that with up to $400-\mu$ l samples 
of homogenate in $0.25 \mathrm{M}$ sucrose. the modified assay can account for virtually all of an added TMP standard if the protocol described above is followed carefully and the blanks and standards are properly corrected by adding the sucrose-containing medium.

The observation that sucrose interferes with a TBA-based assay is not new, although it is apparently easily overlooked. Baumgartner and colleagues (2) showed an interaction between TBA and $30 \mathrm{~mm}$ sucrose $(90 \mu \mathrm{mol}$ in $3 \mathrm{ml}$ ) under different assay conditions, and identified several possibilities about the nature of the chromophore that could be relevant to the conditions that we used. Our observation that there was no strong absorbance peak at $532 \mathrm{~nm}$ in sucrose-containing samples without TMP suggests that malonaldehyde is not one of the major chromophores produced by a reaction involving sucrose. A strong absorbance peak at $440 \mathrm{~nm}$ would indicate the formation of hydroxymethylfurfural due to pyrolysis of the acidic sample (2); we observed strong absorbance of sucrose-containing media at $450 \mathrm{~nm}$, but only in extracted samples. so it is not clear whether this indicates pyrolysis. Although acid treatment and heating are not necessary for reaction between TBA and malonaldehyde, and some investigators have noted that they are not necessary for maximum color development (11), heating reactants to temperatures as high as $100^{\circ} \mathrm{C}$ is common to many protocols. Ohkawa and colleagues (9), in their careful evaluation of the TBA reaction and attempt to optimize the assay conditions, specifically indicated that heating at $95^{\circ} \mathrm{C}$ for $60 \mathrm{~min}$ was not only suitable but necessary for optimum color development.

Buttkus and Bose (7) noted that malonaldehyde can react with nonaldehydic compounds to underestimate the amount of malonaldehyde present in a biolngical sample. However, under the conditions that we used either sucrose itself, a contaminant, or reaction products of sucrose with other compounds clearly cause overestimation of the content of
TBA-reactive substances. Baumgartner and colleagues (2) and Marcuse and Johansson (6) noted the marked ability of TBA to react with aldehydes, but under some assay conditions this may not affect the determination of malonaldehyde in biologic samples. Sucrose plus acetaldehyde in the absence of TMP produces a product that absorbs intensely at $532 \mathrm{~nm}$, corresponding precisely with the absorbance peak for malonaldehyde. We did not find such a peak in the TMP-free solutions, and so the sucrose-acetaldehyde reaction is not likely to have occurred in our experiments. In our samples some of the color produced is likely to be a decomposition product of TBA (11) which strongly absorbs around $450 \mathrm{~nm}$.

Nlthough the interfering substance may be a contaminant in the sucrose, we used an analytical grade that would routinely be selected for in vitro biological assays in which solution volumes are often so large that the use of more highly purified grades would be prohihitively expensive.

\section{REFERENCES}

1. Buege, J. A., and Aust, S. D. (1978) in Methods in Enzymology (Fleischer, S. and Packer, L., eds.) Vol. 52. pp. 302-310, Academic Press. New York.

2. Baumgartner. W. A., Baker, N., Hill, V. A., and Wright, E. T. (1975) Lipids 10, 309-311.

3. Gutteridge, J. M. C., and Tinker, T. R. (1978) Biochem. Med. 19, 127-132.

4. Bird, R. P., Hung, S. O. Hadley, M.. and Draper, H. H. (1983) Anal. Biochem. 128, 240-244.

5. Gray, J. I. (1978) J. Amer. Oil Chem. Soc. 55, 539546.

6. Marcuse, R., and Johansson. L. (1973) J. Amer. On Chem. Soc: 50, 387-391.

7. Buttkus, H., and Bose, R. J. (1972) J. Amer. Oil Chem. Soc: 47, 440-443.

8. Ohkawa, H., Ohishi, N.. and Yagi, K. (1978) J Lipid Res. 19, 1053-1057.

9. Ohkawa, H., Ohishi, N., and Yagi. K. (1979) Anal. Biochem. 95, 351-358.

10. Dahle, L. K., Hill, E. G., and Holman, R. T. (1962) Arch. Biochem. Biophys. 98, 253-261.

11. Tarladgis, B. G., Pearson, A. M., and Dugan, L. R., Jr. (1964) J. Sci. Fd Agric. 15, 602-607.

12. Mihara, M., Uchiyama, M., and Fukuzawa, K. (1980) Biochem. Med 23, 302-311. 